\title{
The Association Between Sleep Disorders and the Risk of Colorectal Cancer in Patients: A Population-based Nested Case-Control Study
}

\author{
CHIA-LING LIN ${ }^{1}$, TA-CHUN LIU ${ }^{2}$, YA-NI WANG ${ }^{3}$, CHI-HSIANG CHUNG ${ }^{4,5}$ and WU-CHIEN CHIEN ${ }^{6,7}$ \\ ${ }^{1}$ Department of Nursing, Chang Gung University of Science and Technology, Taoyuan, Taiwan, R.O.C.; \\ ${ }^{2}$ Department of Molecular Oncology, Medical Science Division, University of Oxford, Oxford, U.K.; \\ ${ }^{3}$ Department of Nursing, Chang Gung University of Science and Technology, Taoyuan, Taiwan, R.O.C.; \\ ${ }^{4}$ School of Public Health, National Defense Medical Center, Taipei, Taiwan, R.O.C.; \\ ${ }^{5}$ Taiwanese Injury Prevention and Safety Promotion Association (TIPSPA), Taipei, Taiwan, R.O.C.; \\ ${ }^{6}$ Department of Medical Research, Tri-Service General Hospital and National, \\ Defense Medical Center, Taipei, Taiwan, R.O.C.; \\ ${ }^{7}$ School of Public Health, National Defense Medical Center, Taipei, Taiwan, R.O.C.
}

\begin{abstract}
Aim: To investigate the risk of colorectal cancer in patients with sleep disorders. Materials and Methods: We identified 7,355 participants with colorectal cancer between January 1, 2000, and December 31, 2013, from the Longitudinal Health Insurance Database 2005 of the Taiwan National Health Insurance Research Database; 29,420 controls were also identified from the same database based on frequency matching on age, sex, and index date of the cases. Diagnoses of sleep disorders by International Classification of Diseases, Ninth Revision, Clinical Modification (ICD-9-CM) in the cases and controls prior to the index date were assessed. The risk of colorectal cancer in patients with sleep disorders was estimated with multivariate logistic regression analyses. Results: The mean age of the 36,775 patients was 63.05 years, and $56 \%$ of them were males. The risk of colorectal cancer was higher in patients with sleep disorders compared to those without [adjusted odds ratio $(O R)=1.29,95 \%$ confidence interval $(C I)=1.13-1.47]$. The risk of colorectal cancer was higher in patients having sleep disorders with depression compared to those without the condition (adjusted OR=5.69, 95\%
\end{abstract}

This article is freely accessible online.

Correspondence to: Wu-Chien Chien, No.325, Section 2, ChengKung Road, Neihu District, Taipei City114, Taiwan, R.O.C. Tel: +886 287923311 ext 19189, Fax: +886 287927235, e-mail: chienwu@ndmctsgh.edu.tw

Key Words: Colorectal cancer, sleep disorders, nested case-control study.
CI=4.01-6.98). Conclusion: The risk of colorectal cancer in patients with sleep disorders was found to be significantly higher by case-control study and particularly pronounced among those with sleep disorders with depression, exhibiting a joint effect on colorectal cancer risk.

Colorectal cancer (CRC) is the most common cancer in the adult population worldwide (1). The Ministry of Health and Welfare in Taiwan reported CRC as the most commonly diagnosed cancer. Mortality rates have increased rapidly and the age-standardized incidence rate was 34.0 per 100,000 in the year of 2002 and 43.0 in in the year of 2015 in Taiwan (2).

The risks of CRC have been extensively examined in the context of diet behavior, physical activity, and obesity $(3,4)$. In recent decades, an increasing number of studies have provided evidence that sleep duration is correlated with the prevalence and incidence of developing CRC. Thompson et $a l$. found that shorter duration of sleep significantly increases risk of colorectal adenomas (5). Another study revealed that longer sleep duration was associated with an increased risk of CRC progressing among individuals who were overweight or snored regularly (6). Lu et al. investigated the data from three cohort studies by a meta-analysis approach and suggested that long sleep duration increased risk of CRC, and the mechanism remains unclear (7).

Sleep is understood to play a pivotal role in human health, including the association of sleep loss with dysregulation of immune system function (8). Sleep deprivation is associated with increasing insulin resistance and decreasing insulin sensitivity (9). Previous studies have extensively investigated the association between the risk of sleep disorders and chronic diseases, such as type 2 diabetes (10), hypertension 


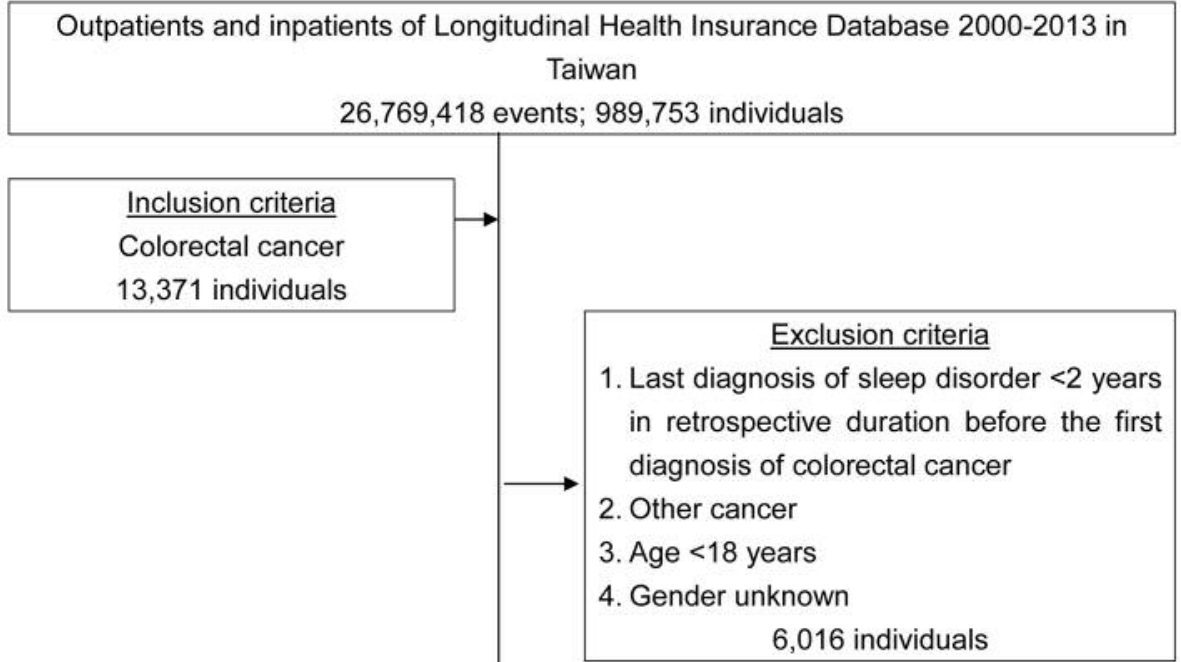

1. Without cancer in study period

2. The same exclusion criteria of case group

3. 4-Fold propensity score matching by gender, age, and index date

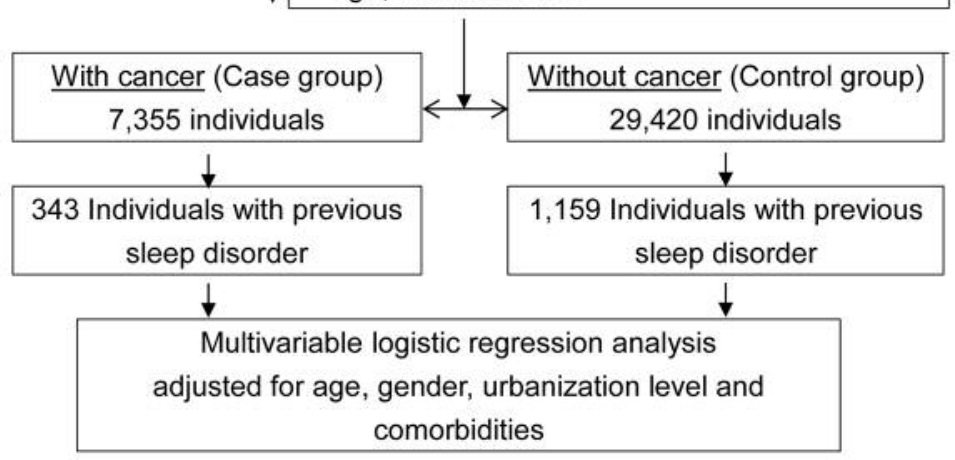

Figure 1. The flowchart of our study design (nested case-control study) from the National Health Insurance Research Database in Taiwan.

(11), and coronary arterial diseases (12). Recently, emerging evidence suggests that sleep disturbances may also increase the risk of several types of cancer, such as of the breast (13), liver (14), and prostate (15). However, longitudinal observational studies surveying the associations between sleep disorders and CRC are limited. Therefore, the aim of this study was to evaluate the risk of CRC incidence among patients with sleep disorders, using a nationwide, health claims research database.

\section{Materials and Methods}

Data source. Taiwan's National Health Insurance program launched a single-payer system on March 1, 1995. As of 2017, 99.9\% of Taiwan's population was enrolled. In this study, the data were collected from the Longitudinal Health Insurance Database 2005 (LHID2005), a subset of National Health Insurance Research
Database 1,000,000 people among the entire population were randomly selected. National Health Research Institutes encrypted all personal identification before the release of LHID2005 to protect patient privacy. In the LHID2005 dossier, the disease diagnosis codes are based on the International Classification of Diseases, Ninth Revision, Clinical Modification (ICD-9-CM) (16). The Ethics Review Board of Tri-Service General Hospital, National Defense Medical Center (TSGHIRB No.1-106-05-169), approved this study.

Identification of case and controls. Patients were defined as incident CRC cases if they had diagnoses of ICD9-CM codes for CRC (ICD9 CM 153-154) in the period between 2000 and 2013. Controls were patients without CRC, frequency matched by sex, age group, and index date, with a control-to-case ratio of 4:1 (Figure 1).

Identification of sleep disorders and comorbidity. The risk factors explored in this study were sleep disorders, which were defined with the criteria of having at least three outpatient diagnoses identified 
Table I. Characteristics of study patients.

\begin{tabular}{|c|c|c|c|c|c|c|c|}
\hline \multirow[b]{2}{*}{ Variable } & \multicolumn{2}{|c|}{ Total } & \multicolumn{2}{|c|}{ Cases } & \multicolumn{2}{|c|}{ Controls } & \multirow[b]{2}{*}{$p$-Value* } \\
\hline & $\mathrm{n}$ & $\%$ & $\mathrm{n}$ & $\%$ & $\mathrm{n}$ & $\%$ & \\
\hline Total & 36,775 & & 7,355 & 20.00 & 29,420 & 80.00 & \\
\hline Gender & & & & & & & 0.999 \\
\hline Male & 20,675 & 56.22 & 4,135 & 56.22 & 16,540 & 56.22 & \\
\hline Female & 16,100 & 43.78 & 3,220 & 43.78 & 12,880 & 43.78 & \\
\hline Age $($ mean \pm SD), years & $63.05 \pm 14.52$ & $63.07 \pm 17.04$ & $63.05 \pm 13.82$ & 0.916 & & & \\
\hline Age group, years & & & & & & & 0.999 \\
\hline $18-40$ & 2,655 & 7.22 & 531 & 7.22 & 2,124 & 7.22 & \\
\hline $45-64$ & 11,980 & 32.58 & 2,396 & 32.58 & 9,584 & 32.58 & \\
\hline$\geq 65$ & 22,140 & 60.20 & 4,428 & 60.20 & 17,712 & 60.20 & \\
\hline \multicolumn{8}{|l|}{ Comorbidities } \\
\hline $\mathrm{T} 2 \mathrm{DM}$ & 7,759 & 21.10 & 1,589 & 21.60 & 6,170 & 20.97 & 0.235 \\
\hline Hypertension & 6,310 & 17.16 & 1,291 & 17.55 & 5,019 & 17.06 & 0.316 \\
\hline Depression & 564 & 1.53 & 235 & 3.20 & 329 & 1.12 & $<0.001$ \\
\hline Stroke & 1,101 & 2.99 & 228 & 3.10 & 873 & 2.97 & 0.551 \\
\hline Dementia & 680 & 1.85 & 127 & 1.73 & 553 & 1.88 & 0.384 \\
\hline CKD & 586 & 1.59 & 109 & 1.48 & 477 & 1.62 & 0.434 \\
\hline Urbanization level & & & & & & & $<0.001$ \\
\hline 1 (Highest) & 11,456 & 31.15 & 2,725 & 37.05 & 8,731 & 29.68 & \\
\hline 2 & 16,546 & 44.99 & 3,362 & 45.71 & 13,184 & 44.81 & \\
\hline 3 & 2,671 & 7.26 & 360 & 4.89 & 2,311 & 7.86 & \\
\hline 4 (Lowest) & 6,102 & 16.59 & 908 & 12.35 & 5,194 & 17.65 & \\
\hline Sleep disorder & 1,502 & & 343 & 4.66 & 1159 & 3.94 & $<0.001$ \\
\hline
\end{tabular}

T2DM, Type 2 diabetes; CKD, chronic kidney diseases. ${ }^{*}$ Chi-square/Fisher exact test on category variables and $t$-test on continuous variables.

with the ICD-9 code. These codes included 780.5 (sleep disturbance), 780.50 (sleep disturbance, unspecified), 780.52 (insomnia, unspecified), 780.51, 780.53, 780.57 (sleep apnea syndrome), 307.4 (specific disorders of sleep of nonorganic origin), 780.54 (hypersomnia, unspecified), 780.55 (disruptions of 24-h sleep-wake cycle, unspecified), 780.56 (dysfunctions associated with sleep stages or arousal from sleep), 780.58 (sleep-related movement disorder, unspecified), 780.59 (sleep disturbance, other) in the period between 2000 and 2013.

The comorbidities evaluated in this study included type 2 diabetes (ICD-9-CM 250), hypertension (ICD-9-CM 401-405), depression (ICD-9-CM 296.2-296.3, 300.4, 311), stroke (ICD-9-CM 430-438), dementia (ICD-9-CM 290, 294.1, 331.0) and chronic kidney disease (ICD-9-CM 585).

Statistical analysis. Descriptive statistics were used for characteristic information, including the percentage, mean, and standard deviation. Chi-squared and $t$-tests were used to evaluate the distributions of categorical and continuous variables between case and controls. The incidence densities of CRC were calculated according to age, sex, comorbidities, and urbanization level. Unconditional multiple logistic regression analyses were performed to evaluate the risks of CRC associated with sleep disorders with and without adjusting for age, sex, and comorbidities, respectively. Adjusted models with significant covariates were constructed through backward selection using the likelihood ratio test. Subgroup analyses were conducted with stratification by different types of sleep disorder. All analyses were performed using SPSS version 21
(IBM, Armonk, NY, USA). A $p$-value of less than 0.05 was considered significant.

\section{Results}

Demographic data. Table I shows the demographic characteristics of patients with and without CRC from 2000 to 2013 . The mean age of the total 36,775 patients was $63.05 \pm 14.52$ years and $56.22 \%$ of them were males. Age and sex were frequency-matched between the case and control groups to ensure no significant differences in the distributions of the two variables existed. Compared with those in the control group, the comorbidity of depression was more prevalent in the case group (Table I). Among 7,355 patients with $\mathrm{CRC}$, the proportion of those with sleep disorders was $4.66 \%(343 / 7,355)$, while $3.94 \%(1,159 / 29,420)$ of the control group had a sleep disorder $(p<0.001)$.

Factors of CRC using logistic regression. Table II shows participants aged $45-64$ years, or $\geq 65$ years [adjusted odds ratio $(A O R)=1.51 ; 95 \%$ confidence interval $(\mathrm{CI})=1.22-1.88$; $\mathrm{AOR}=1.88, \quad 95 \% \quad \mathrm{CI}=1.52-2.33$, respectively $]$ had significantly higher risk of CRC compared with those aged 18-40 years (controls). There was also a significantly higher risk of cancer for patients with comorbidities (AOR: type 2 
diabetes $=1.15 ; 95 \% \mathrm{CI}=1.01-1.31 ;$ depression $=3.48,95 \%$ $\mathrm{CI}=2.51-4.82 ; \quad$ stroke $=1.21,95 \% \mathrm{CI}=1.01-1.43$, chronic kidney disease $=2.01,95 \% \mathrm{CI}=1.50-2.71$, respectively) compared with those in the control groups.

Risk of CRC stratified by sleep disorder subgroups using logistic regression. Table III shows a 1.29-times higher risk of $\mathrm{CRC}$ in patients with sleep disorders compared to those in the control group $(\mathrm{AOR}=1.29,95 \% \mathrm{CI}=1.13-1.47)$. Multivariate logistic regression analyses revealed that in the case of CRC, those previously diagnosed with sleep apnea or insomnia had a higher risk of developing CRC $(\mathrm{AOR}=1.76,95 \% \mathrm{CI}=1.54-$ 2.00; $\mathrm{AOR}=1.54,95 \% \mathrm{CI}=1.35-1.75)$. In addition, patients with sleep disorders and depression had a significantly higher risk of developing CRC compared with those without these conditions $(\mathrm{AOR}=5.69 ; 95 \% \mathrm{CI}=4.01-6.98)$ (Table IV).

\section{Discussion}

The present study found that sleep disorders were significantly associated with a higher risk of CRC, particularly in those individuals with depression. These results suggest that sleep disorders may be a novel risk factor for CRC. Moreover, when a patient suffered both sleep disorders and depression, the risk of CRC was 5.69-times higher compared with the control group. This reflects a joint effect between sleep disorders and depression on increasing the risk of CRC. To the best of our knowledge, this is the first retrospective case-control study examining the association between sleep disorders and the risk of CRC.

Although the physiological mechanisms remain unclear of the association between sleep disorders and CRC, we inferred a potential mechanism from previous studies that may provide some insights into our observation. Some evidence supports the notion that the disruption of circadian rhythm is involved in the decreased production of melatonin and interference in the stability of clock genes, which could potentially promote the development of cancer (17-19). Previous study indicated that the mechanisms of action of circadian genes in cancer include the down-regulation of PER2 and the up-regulation of $\beta$-catenin protein levels (19), which causes proliferation of colon cancer cells and the formation of intestinal and colonic polyps. This suggests that the disruption of the peripheral intestinal circadian clock may contribute to intestinal epithelial neoplastic transformation of human CRC (20). Circadian disruption is classified as "probably carcinogenic" to humans by the International Agency for Research on Cancer (IARC) (21).

Melatonin is a natural hormone linked to DNA repair. Reiter has suggested mechanisms of cancer inhibition through melatonin (17). Previous evidence has also supported melatonin as restraining cancer growth and increasing immune function (18). Sleep disruption may
Table II. Logistic regression of colorectal cancer variables.

\begin{tabular}{|c|c|c|c|c|}
\hline Variable & AOR & $95 \% \mathrm{CI}$ & $95 \% \mathrm{CI}$ & $p$-Value \\
\hline \multicolumn{5}{|l|}{ Gender } \\
\hline Male & 1.06 & 0.95 & 1.17 & 0.316 \\
\hline Female & Reference & & & \\
\hline \multicolumn{5}{|c|}{ Age group, years } \\
\hline $18-40$ & Reference & & & \\
\hline $45-64$ & 1.51 & 1.22 & 1.88 & $<0.001$ \\
\hline$\geq 65$ & 1.88 & 1.52 & 2.33 & $<0.001$ \\
\hline \multicolumn{5}{|l|}{$\mathrm{T} 2 \mathrm{DM}$} \\
\hline Without & Reference & & & \\
\hline With & 1.15 & 1.01 & 1.31 & 0.030 \\
\hline \multicolumn{5}{|l|}{ Hypertension } \\
\hline Without & Reference & & & \\
\hline With & 0.95 & 0.84 & 1.08 & 0.428 \\
\hline \multicolumn{5}{|l|}{ Depression } \\
\hline Without & Reference & & & \\
\hline With & 3.48 & 2.51 & 4.82 & $<0.001$ \\
\hline \multicolumn{5}{|l|}{ Stroke } \\
\hline Without & Reference & & & \\
\hline With & 1.21 & 1.01 & 1.43 & 0.035 \\
\hline \multicolumn{5}{|l|}{ Dementia } \\
\hline Without & Reference & & & \\
\hline With & 1.31 & 0.85 & 2.01 & 0.221 \\
\hline \multicolumn{5}{|l|}{ CKD } \\
\hline Without & Reference & & & \\
\hline With & 2.01 & 1.50 & 2.71 & $<0.001$ \\
\hline \multicolumn{5}{|c|}{ Urbanization level } \\
\hline 1 (Highest) & 0.80 & 0.68 & 0.94 & 0.007 \\
\hline 2 & 0.85 & 0.73 & 0.98 & 0.024 \\
\hline 3 & 0.74 & 0.59 & 0.93 & 0.009 \\
\hline 4 (Lowest) & Reference & & & \\
\hline
\end{tabular}

T2DM, Type 2 diabetes; HTN, hypertension; CKD, chronic kidney diseases; AOR, odds ratio adjusted for variables listed in the table, CI, confidence intervaI.

affect the secretion of melatonin and is associated with decrease in the function of removing free radicals and protecting against oxidative DNA damage $(20,22)$.

Inadequate sleep may reduce the release of immunestimulating hormones, such as growth hormone, prolactin, and dopamine, which may affect the natural state of the immune system (23). Impaired sleep was shown to have an effect on the performance of pro-inflammatory cytokine genes including interleukin-6 (IL6) and tumor necrosis factor- $\alpha$ (TNFA) (24). Activation of cellular and genomic markers of inflammation may also contribute to the development of CRC (25).

Sleep deprivation increases the sensitivity to dextran sodium sulfate (DSS) shown to induce colitis in animal studies (26). An epidemiological study also indicated that sleep of less than 6 hours or a more than 9 hours per day is associated with an increased risk of ulcerative colitis (27). The association between ulcerative colitis and CRC is also 
Lin et al: Sleep Disorders and Risk of CRC

Table III. Factors of colorectal cancer stratified by sleep disorder subgroups using logistic regression.

\begin{tabular}{lcccccccccc}
\hline & \multicolumn{3}{c}{ With sleep disorder } & \multicolumn{3}{c}{ Without sleep disorder } & \multicolumn{3}{c}{ With vs. without } \\
\cline { 2 - 11 } Subgroup & Exposure & Person-years & Rate* & Exposure & Person-years & Rate* & Ratio & AOR & $95 \%$ CI & $p$-Value \\
\hline Total & 343 & $20,208.18$ & 16.97 & 1,159 & $165,801.05$ & 6.99 & 2.43 & 1.29 & $1.13-1.47$ & $<0.001$ \\
Sleep apnea & 27 & $20,208.18$ & 1.34 & 67 & $165,801.05$ & 0.40 & 3.35 & 1.76 & $1.54-2.00$ & $<0.001$ \\
Insomnia & 233 & $20,208.18$ & 11.53 & 660 & $165,801.05$ & 3.98 & 2.90 & 1.54 & $1.35-1.75$ & $<0.001$ \\
Other & 109 & $20,208.18$ & 5.39 & 468 & $165,801.05$ & 2.82 & 1.91 & 1.02 & $0.89-1.16$ & 0.183 \\
\hline
\end{tabular}

AOR, Odds ratio adjusted for the variables listed in Table II; CI, confidence interval. ICD-9-CM code: Sleep apnea: 780.51, 780.53, and 780.57; insomnia: 780.52; other: $307.4,780.50,780.54-780.56$, and 780.59 . *Per $10^{3}$ person-years.

acknowledged by Yashiro et al. (28). The abovementioned pathophysiological factors may explain the association between sleep disorders and CRC shown in this study.

This study found a higher risk of CRC compared to the reference group in two subgroups of patients with sleep disorder, sleep apnea and insomnia. A previous study demonstrated a heightened risk for cancer development in individuals suffering from insomnia, parasomnia or obstructive sleep apnea (15). Another study showed that a short sleep duration is associated with a higher risk of CRC development $(5,6)$. Moreover, a longer sleep duration $(\geq 9$ hours/per night) is also associated with an increased risk for developing CRC among individuals who snored or overweight (6). Zhang et al. raises the possibility that obstructive sleep apnea and intermittent hypoxemia may contribute to cancer risk (6). Furthermore, intermittent hypoxia and sleep fragmentation caused by obstructive sleep apnea was associated with a poorer prognosis or higher incidence of cancer (29). Further studies are required to investigate sleep problems as a modifiable risk factor in the context of the progression of CRC.

Our findings revealed that the risk for CRC in patients with both sleep disorder and depression was significantly higher (5.69-fold) than for patients without these conditions (sleep disorders 1.29-fold, depression 3.48-fold). Therefore, the joint effect of sleep disorder and depression is in need of attention. To our knowledge, this is the first longitudinal population-based study on the risk of CRC associated with sleep disorders combined with depression. Determining the mechanism explaining the observed association was not the aim of this study. Prior evidence supported a bidirectional association between sleep disorders and depression (30). Depression is suggested to suppress the immune system causing an elevation of IL6 (31) and an increased concentration of C-reactive protein (32), each of which has been associated with an increased risk of CRC $(32,33)$. On the contrary, the Nurses' Health Study indicated depressive symptoms were unrelated to the risk of colorectal adenomas
Table IV. Colorectal cancer risk stratified by sleep disorders and depression using logistic regression.

\begin{tabular}{lcccc}
\hline Sleep disorder & Depression & AOR & $95 \%$ CI & $p$-Value \\
\hline Without & Without & Reference $(1.00)$ & & \\
With & Without & 1.29 & $1.13-1.47$ & $<0.001$ \\
Without & With & 3.48 & $2.50-4.83$ & $<0.001$ \\
With & With & 5.69 & $4.01-6.98$ & $<0.001$ \\
\hline
\end{tabular}

AOR, Odds ratio adjusted for the variables listed in Table II; CI, confidence interval. Joint effect (interaction term): colorectal cancer $\times$ depression, $p<0.001$.

(34). We understand that factors involving CRC are complex combinations of risks and interactions among multiple variables, further suggesting that the confirmed associations were independent of these possible pathways. It is important for further studies to clarify the direct or indirect effects of sleep disorders and depression on the relationship to CRC.

There are several potential limitations of the present study. Firstly, the National Health Insurance Research Database does not provide detailed information, such as physical activity, alcohol consumption, tobacco use and diet behaviors, which may have resulted in residual confounding. Secondly, body mass index and obesity were not variables included in our study. It is crucial to note that a previous study reported obesity having a direct and independent relationship with CRC, suggesting an association between obesity and CRC (4). We suggest that future studies should consider investigating the effect of obesity, and sleep disorders on CRC. Thirdly, despite the meticulous design of this study and its control of confounding factors, biases might remain from possibly unmeasured or unknown confounding factors, such as the onset of depression, stage of CRC at diagnosis and medication that might have influenced the results. A prospective cohort study is suggested in order to evaluate the relationship between sleep disorders and CRC. 


\section{Conclusion}

The present study revealed that sleep disorders are associated with an increased prevalence of CRC. Both sleep disorders and depression of patients have a synergistic effect on CRC, compared to the reference group. Sleep disorder might be a new risk factor for the development of CRC. Healthcare providers should, perhaps, pay more attention to the association between chronic sleep problems and the risk of developing CRC.

\section{Conflicts of Interests}

This study was performed without financial support and there are no conflicts of interest to declare.

\section{Acknowledgements}

This study was supported by Grants from Tri-service Hospital Research Foundation (TSGH- C108-003), and the sponsor had no role in study design, data collection and analysis, or description of the manuscript. The Authors thank the National Health Research Institute of Taiwan for providing the insurance claims data.

\section{References}

1 Stewart B and Wild CP: World cancer report 2014. World Health Organization 2017.

2 The Epidemiology of Colorectal Cancer in Taiwan. Health Promotion Administration, Ministry of Health and Welfare in Taiwan, 2017. https://www.hpa.gov.tw/Pages/Detail.aspx? nodeid $=615 \&$ pid $=1126$.

3 Giovannucci E, Ascherio A, Rimm EB, Colditz GA, Stampfer MJ and Willett WC: Physical activity, obesity, and risk for colon cancer and adenoma in men. Ann Intern Med 122(5): 327-334, 1995.

4 Moghaddam AA, Woodward M and Huxley R: Obesity and risk of colorectal cancer: a meta-analysis of 31 studies with 70,000 events. Cancer Epidemiol Biomarkers Prev 16(12): 2533-2547, 2007.

5 Thompson CL, Larkin EK, Patel S, Berger NA, Redline S and Li L: Short duration of sleep increases risk of colorectal adenoma. Cancer 117(4): 841-847, 2011.

6 Zhang X, Giovannucci EL, Wu K, Gao X, Hu F, Ogino S, Schernhammer ES, Fuchs CS, Redline S, Willett WC and Ma J: Associations of self-reported sleep duration and snoring with colorectal cancer risk in men and women. Sleep 36(5): 681-688, 2013.

7 Lu Y, Tian N, Yin J, Shi Y and Huang Z: Association between sleep duration and cancer risk: a meta-analysis of prospective cohort studies. PLoS One 8(9): e74723, 2013.

8 Irwin MR, Wang M, Ribeiro D, Cho HJ, Olmstead R, Breen EC, Martinez-Maza $\mathrm{O}$ and Cole S: Sleep loss activates cellular inflammatory signaling. Biol Psychiatry 64(6): 538-540, 2008.

9 Spiegel K, Knutson K, Leproult R, Tasali E and Van Cauter E: Sleep loss: a novel risk factor for insulin resistance and Type 2 diabetes. J Appl Physiol (1985) 99(5): 2008-2019, 2005.
10 Lin CL, Chien WC, Chung CH and Wu FL: Risk of type 2 diabetes in patients with insomnia: A population-based historical cohort study. Diabetes Metab Res Rev 34(1): e2930, 2018.

11 Lin C, Liu T, Lin F, Chung CH and Chien WC: Association between sleep disorders and hypertension in Taiwan: a nationwide population-based retrospective cohort study. J Hum Hypertens 31(3): 220-224, 2017.

12 Hsu CY, Chen YT, Chen MH, Huang CC, Chiang CH, Huang PH, Chen JW, Chen TJ, Lin SJ, Leu HB and Chan WL: The association between insomnia and increased future cardiovascular events: a nationwide population-based study. Psychosom Med 77(7): 743-751, 2015.

13 Davis S, Mirick DK and Stevens RG: Night shift work, light at night, and risk of breast cancer. J Natl Cancer Inst 93(20): 15571562, 2001.

14 Hu LY, Chen PM, Hu YW, Shen CC, Perng CL, Su TP, Yen SH, Tzeng CH, Chiou TJ, Yeh CM, Chen TJ, Wang WS and Liu CJ: The risk of cancer among patients with sleep disturbance: a nationwide retrospective study in Taiwan. Ann Epidemiol 23(12): 757-761, 2013.

15 Fang HF, Miao NF, Chen CD, Sithole T and Chung MH: Risk of cancer in patients with insomnia, parasomnia, and obstructive sleep apnea: a nationwide nested case-control study. J Cancer 6(11): 1140-1147, 2015

16 Slee VN: The International Classification of Diseases: ninth revision (ICD-9), Ann Intern Med vol. 88, 1978.

17 Reiter RJ: Mechanisms of cancer inhibition by melatonin. J Pineal Res 37(3): 213-214, 2004.

18 Martínez-García MÁ, Campos-Rodríguez F and Almendros I: Sleep disorders and cancer. Curr Sleep Medicine Rep 2(1): 1-11, 2016.

19 Wood PA, Xiaoming Yang and Hrushesky WJM: Clock genes and cancer. Integr. Cancer Ther 8: 303-308, 2009.

20 Savvidis C and Koutsilieris M: Circadian rhythm disruption in cancer biology. Mol Med 18: 1249-1260, 2012.

21 Stevens RG, Hansen J, Costa G, Haus E, Kauppinen T, Aronson KJ, Castaño-Vinyals G, Davis S, Frings-Dresen MH, Fritschi L, Kogevinas M, Kogi K, Lie JA, Lowden A, Peplonska B, Pesch B, Pukkala E, Schernhammer E, Travis RC, Vermeulen R, Zheng T, Cogliano V and Straif K: Considerations of circadian impact for defining 'shift work' in cancer studies: IARC Working Group Report. Occup Environ Med 68(2): 154-162, 2011.

22 Sliwinski T, Rozej W, Morawiec-Bajda A, Morawiec Z, Reiter $\mathrm{R}$ and Blasiak $\mathrm{J}$ : Protective action of melatonin against oxidative DNA damage - chemical inactivation versus base-excision repair. Mutat Res 634 (1-2): 220-227, 2007.

23 Lange T, Dimitrov S and Born J: Effects of sleep and circadian rhythm on the human immune system. Ann NY Acad Sci 1193: 48-59, 2010.

24 Irwin MR, Wang M, Campomayor CO, Collado-Hidalgo A and Cole S: Sleep deprivation and activation of morning levels of cellular and genomic markers of inflammation. Arch Intern Med 166(16): 1756-1762, 2006.

25 Itzkowitz SH, Yio X: Inflammation and cancer IV: Colorectal cancer in inflammatory bowel disease: the role of inflammation. Am J Physiol Gastrointest Liver Physiol 287(1): G7-17, 2004.

26 Tang Y, Preuss F, Turek FW, Jakate S and Keshavarzian A: Sleep deprivation worsens inflammation and delays recovery in a mouse model of colitis. Sleep Med 10(6): 597-603, 2009. 
27 Ananthakrishnan AN, Khalili H, Konijeti GG, Higuchi LM, de Silva P, Fuchs CS, Richter JM, Schernhammer ES and Chan AT: Sleep duration affects risk for ulcerative colitis: a prospective cohort study. Clin Gastroenterol Hepatol 12(11): 1879-1886, 2014

28 Yashiro M: Ulcerative colitis-associated colorectal cancer Cancer 117: 841-847, 2011.

29 Almendros I, Montserrat J, Ramirez J, Torres M, Duran-Cantolla J, Navajas D and Farré R: Intermittent hypoxia enhances cancer progression in a mouse model of sleep apnoea. Eur Respir J 39(1): 215-217, 2012.

30 Nutt D, Wilson S, Paterson L: Sleep disorders as core symptoms of depression. Dialogues Clin Neurosci 10(3): 329-336, 2008.

31 Glaser R, Robles TF, Sheridan J, Malarkey WB and KiecoltGlaser JK: Mild depressive symptoms are associated with amplified and prolonged inflammatory responses after influenza virus vaccination in older adults. Arch Gen Psychiatry 60(10): 1009-1014, 2003.
32 Erlinger TP, Platz EA, Rifai N and Helzlsouer KJ: C-Reactive protein and the risk of incident colorectal cancer. JAMA 291(5): 585-590, 2004.

33 Kinoshita T, Ito H and Miki C: Serum interleukin-6 level reflects the tumor proliferative activity in patients with colorectal carcinoma. Cancer 85(12): 2526-2531, 1999.

34 Kroenke CH, Bennett GG, Fuchs C, Giovannucci E, Kawachi I, Schernhammer E, Holmes MD and Kubzansky LD: Depressive symptoms and prospective incidence of colorectal cancer in women. Am J Epidemiol 162(9): 839-848, 2005.

Received November 25, 2018

Revised December 8, 2018

Accepted December 11, 2018 